\title{
Using the NEMA NU 4 PET Image Quality Phantom in Multipinhole Small-Animal SPECT
}

\author{
Anita A. Harteveld ${ }^{1,2}$, Antoi P.W. Meeuwis ${ }^{1}$, Jonathan A. Disselhorst ${ }^{1}$, Cornelis H. Slump ${ }^{2}$, Wim J.G. Oyen ${ }^{1}$, \\ Otto C. Boerman ${ }^{1}$, and Eric P. Visser ${ }^{1}$ \\ ${ }^{1}$ Department of Nuclear Medicine, Radboud University Nijmegen Medical Centre, Nijmegen, The Netherlands; and ${ }^{2}$ Institute for \\ Technical Medicine, University of Twente, Enschede, The Netherlands
}

\begin{abstract}
Several commercial small-animal SPECT scanners using multipinhole collimation are presently available. However, generally accepted standards to characterize the performance of these scanners do not exist. Whereas for small-animal PET, the National Electrical Manufacturers Association (NEMA) NU 4 standards have been defined in 2008 , such standards are still lacking for small-animal SPECT. In this study, the image quality parameters associated with the NEMA NU 4 image quality phantom were determined for a small-animal multipinhole SPECT scanner. Methods: Multiple whole-body scans of the NEMA NU 4 image quality phantom of 1 -h duration were performed in a U-SPECT-II scanner using ${ }^{99 m T c}$ with activities ranging between 8.4 and $78.2 \mathrm{MBq}$. The collimator contained 75 pinholes of $1.0-\mathrm{mm}$ diameter and had a bore diameter of 98 $\mathrm{mm}$. Image quality parameters were determined as a function of average phantom activity, number of iterations, postreconstruction spatial filter, and scatter correction. In addition, a mouse was injected with ${ }^{99 m}$ Tc-hydroxymethylene diphosphonate and was euthanized $6.5 \mathrm{~h}$ after injection. Multiple whole-body scans of this mouse of 1-h duration were acquired for activities ranging between 3.29 and $52.7 \mathrm{MBq}$. Results: An increase in the number of iterations was accompanied by an increase in the recovery coefficients for the small rods $\left(\mathrm{RC}_{\mathrm{rod}}\right)$, an increase in the noise in the uniform phantom region, and a decrease in spillover ratios for the cold-air- and water-filled scatter compartments $\left(\mathrm{SOR}_{\text {air }}\right.$ and $\left.\mathrm{SOR}_{\text {wat }}\right)$. Application of spatial filtering reduced image noise but lowered $\mathrm{RC}_{\text {rod. }}$. Filtering did not influence $\mathrm{SOR}_{\text {air }}$ and $\mathrm{SOR}_{\text {wat }}$. Scatter correction reduced $\mathrm{SOR}_{\text {air }}$ and $\mathrm{SOR}_{\text {wat }}$. The effect of total phantom activity was primarily seen in a reduction of image noise with increasing activity. $\mathrm{RC}_{\text {rod }}, \mathrm{SOR}_{\mathrm{air}}$, and $\mathrm{SOR}_{\text {wat }}$ were more or less constant as a function of phantom activity. The relation between acquisition and reconstruction settings and image quality was confirmed in the $99 \mathrm{~m}$ Tc-hydroxymethylene diphosphonate mouse scans. Conclusion: Although developed for small-animal PET, the NEMA NU 4 image quality phantom was found to be useful for small-animal SPECT as well, allowing for objective determination of image quality parameters and showing the tradeoffs between several of these parameters on variation of acquisition and reconstruction settings.
\end{abstract}

Received Dec. 28, 2010; revision accepted May 18, 2011.

For correspondence contact: Eric P. Visser, Department of Nuclear Medicine (internal postal code 444), Radboud University Nijmegen Medical

Centre, P.O. Box 9101, 6500 HB Nijmegen, The Netherlands.

E-mail: e.visser@nucmed.umcn.n

Published online Aug. 17, 2011.

COPYRIGHT (C 2011 by the Society of Nuclear Medicine, Inc
Key Words: single photon emission computed tomography; small-animal; image quality; NEMA; multi-pinhole

J Nucl Med 2011; 52:1646-1653

DOI: 10.2967/jnumed.110.087114

$\mathbf{S}$

P PECT has become an important noninvasive imaging technique in small-animal research. An overview of the position of small-animal SPECT relative to other imaging techniques has been presented in a review paper by Meikle et al. (1).

Recent developments in small-animal SPECT focused on improvement of the spatial resolution using pinhole collimation. However, high spatial resolution, obtained by small-diameter pinholes, is associated with low sensitivity and increased image noise. To improve the sensitivity, multiple pinholes can be used. Most commercially available small-animal SPECT scanners today (Bioscan NanoSPECT, GE Triumph X-SPECT, MILabs U-SPECT-II (2), and Siemens Inveon SPECT) use multiple-pinhole collimation, although some of them can also be equipped with traditional, lead shield-based collimators (NanoSPECT, X-SPECT, and Inveon).

To quantitatively characterize the performance of smallanimal SPECT scanners, image quality can be measured in a phantom that produces images simulating those obtained in a whole-body (WB) study for a small rodent with hot lesions, as well as uniform hot and some cold areas. For small-animal PET, a standard phantom with corresponding image quality parameters (NU 4 IQ phantom) has been defined by the National Electrical Manufacturers Association (NEMA) NU 42008 standards (3). However, generally accepted standards to characterize small-animal SPECT scanners are lacking, and more specifically, a dedicated phantom with exactly defined image quality parameters does not exist. As of March 2011, no initiative has been taken up yet by NEMA for small-animal SPECT guidelines. Most of the scientific literature on image quality in small-animal SPECT is based on qualitative demonstrations of the effective spatial resolution using Derenzo-like phantoms with hot and cold rods (2,4-9). An anatomic phantom 
to mimic rat brain structures has been described by Beekman et al. (10). Recently, a micro-hollow-sphere phantom based on superposition was developed to avoid the problems associated with nonzero wall thickness (11). Fabrication of phantoms for small-animal imaging using stereolithography has been described by Park et al. (12).

In this study, the NU 4 IQ phantom was used to characterize the image quality performance of a multipinhole small-animal SPECT system with different image reconstruction settings for a range of activity levels, with focus on activity and scan duration typically applied in WB mouse experiments. Phantom results will be demonstrated in an animal experiment.

\section{MATERIALS AND METHODS}

\section{Phantom and Image Quality Parameters}

The NU 4 IQ phantom (3) is composed of a main phantom body that contains a fillable cylindric chamber $30 \mathrm{~mm}$ in diameter and $30 \mathrm{~mm}$ in length and a solid part $20 \mathrm{~mm}$ in length into which 5 fillable rods with diameters of $1,2,3,4$, and $5 \mathrm{~mm}$ have been drilled. A lid attached to the uniform region of the phantom supports 2 cold-region chambers. These regions are hollow cylinders $15 \mathrm{~mm}$ in length and $8 \mathrm{~mm}$ in inner diameter with $1-\mathrm{mm}$ wall thickness, to be filled with nonradioactive water and air, respectively. The phantom was constructed according to the NEMA NU 4 specifications by Agile Engineering. The fillable rods of different diameters are used to determine the activity recovery coefficients, which are indicative of spatial resolution. The relative standard deviation in a uniform phantom region is a measure of the signal-to-noise ratio, whereas the overall uniformity in this region characterizes the attenuation and scatter correction performance. The activity measured in nonradioactive water- and air-filled compartments is indicative of the spillover and scatter correction performance.

The following NU 4 IQ parameters were determined: image noise, expressed as the percentage standard deviation $\left(\% \mathrm{STD}_{\text {unif }}\right)$ in a central, cylindric volume of interest over the center of the uniform region of the phantom; activity recovery coefficients for the filled rods $\left(\mathrm{RC}_{\mathrm{rod}}\right)$, expressed as the measured activity concentration in the rods divided by the mean phantom concentration; their percentage standard deviation $\left(\% \mathrm{STD}_{\mathrm{RC}}\right)$; and spillover ratios for the nonradioactive water- and air-filled compartments $\left(\mathrm{SOR}_{\mathrm{w}}\right.$ at and $\mathrm{SOR}$ air, respectively), defined as the activity concentration measured in these compartments divided by the mean phantom concentration. Exact definitions and phantom locations where these parameters were determined have been described elsewhere (3).

An additional parameter that was determined is the effective WB sensitivity, defined as the average photopeak counting rate over a WB scan covering the phantom, divided by the average activity in the phantom during the scan. In addition to centered point source sensitivity, as used in small-animal PET with a generally fixed field of view, effective WB sensitivity could be an interesting parameter since in multipinhole SPECT the sensitivity critically depends on the size of the scanned object. The volume to be scanned determines, for instance, the radii of rotation in case of rotating collimators and the number of transaxial bed positions needed for complete coverage of the object in case of cylindric, static collimators using scanning-focus mode (2). Moreover, an extended source is expected to more realistically reflect the effects of scatter and attenuation as encountered in WB scans than does a centered point source.

\section{Acquisition Settings}

The NU 4 IQ phantom was filled with an aqueous solution of ${ }^{99 \mathrm{~m}} \mathrm{Tc}$ and scanned in a U-SPECT-II scanner (2) in WB scanningfocus mode using a collimator with 75 pinholes of 1.0-mm diameter and a bore diameter of $98 \mathrm{~mm}$. In total, 20 consecutive WB scans of 1.01997-h each were acquired. The data acquisition time per WB scan was $1 \mathrm{~h}$, with the additional $1 \mathrm{~min}$ and $12 \mathrm{~s}$ being attributed to bed motion and the start-up time of the data acquisition for each new bed position. Each individual WB scan consisted of 36 bed positions necessary to scan the entire phantom. The axial bed travel range was $81.7 \mathrm{~mm}$.

Total activity and activity concentration at the start of the first WB scan were $82.8 \mathrm{MBq}$ and $4.01 \mathrm{MBq} / \mathrm{mL}$, respectively, which by physical decay were reduced to $7.90 \mathrm{MBq}$ and $0.38 \mathrm{MBq} / \mathrm{mL}$ at the end of the last WB scan. The activity was measured in a dose calibrator (VDC 404; Veenstra) with an accuracy of $3 \%$.

\section{Reconstruction Settings}

The list-mode data of the $20 \mathrm{WB}$ scans with different activities were reconstructed using the statistical iterative algorithm (13-15) as implemented in the U-SPECT-II system software version 34i3, into image matrices of $123 \times 131 \times 259$ pixels of $0.375 \mathrm{~mm}$ in all directions, resulting in image sizes of $46 \times 49 \times 97 \mathrm{~mm}^{3}$. The photopeak window was $126-154 \mathrm{keV}$, corresponding to a width of $20 \%$ of the peak energy $(140 \mathrm{keV})$. Because there are no official recommendations by the manufacturer, multiple reconstructions were performed with different settings to evaluate their effect on the image quality parameters. The number of iterations was varied between 1 and 6, with the number of subsets kept fixed at 16 . Reconstructions were performed without and with scatter correction using the triple-energy-window method as described by Ogawa et al. (16) and implemented in the U-SPECT-II vendor software. The scatter windows were $100-120$ and $160-180 \mathrm{keV}$, applied with a weight factor of 0.7 to match the width of the photopeak window. The upper energy window was included for scatter correction, since nonzero signal was measured in this window. The effect of postreconstruction spatial filtering was evaluated using no filtering and 3-dimensional gaussian filters with kernel widths of 1.0 and $1.5 \mathrm{~mm}$ in full width at half maximum.

\section{Animal Study}

A BALB/c nude mouse was injected with $239 \mathrm{MBq}$ of ${ }^{99 \mathrm{~m}} \mathrm{Tc}-$ hydroxymethylene diphosphonate and scanned $6.5 \mathrm{~h}$ after injection in WB scanning-focus mode, again using the collimator with 1.0-mm-diameter pinholes and a bore diameter of $98 \mathrm{~mm}$. The mouse was euthanized $6.3 \mathrm{~h}$ after injection to allow maximal uptake in the bone structures. Just before scanning, the euthanized mouse was placed in the dose calibrator. The measured activity was $52.7 \mathrm{MBq}$, which, because of photon attenuation, is of course a slight underestimation of the total-mouse activity.

In total, 24 consecutive WB scans of $1.0036 \mathrm{~h}$ each were acquired, leading to a total scan time of $24.0875 \mathrm{~h}$. The data acquisition time per WB mouse scan was $1 \mathrm{~h}$, with the additional time of approximately $13 \mathrm{~s}$ being attributed to bed motion and the start-up time of the data acquisition for each new bed position. Each WB scan consisted of 6 bed positions necessary to scan the total mouse. Because the mouse was euthanized and biologic clearance was absent, the activity for each scan could be calculated on the basis of physical decay. This calculation led to 
activities ranging between $52.7 \mathrm{MBq}$ at the start of the first scan and $3.29 \mathrm{MBq}$ at the end of the last scan (again neglecting the effect of photon attenuation).

The list-mode data of the 24 time frames have been reconstructed into image matrices of $70 \times 64 \times 254$ pixels of $0.375 \mathrm{~mm}$ in all directions, resulting in image sizes of $26 \times 24 \times 95 \mathrm{~mm}^{3}$; that is, pixel sizes were equal to those of the phantom scans, but matrix sizes differed because of different object sizes. The same variation of reconstruction settings as in the phantom experiment was applied to compare their effects on phantom image quality parameters with the direct observations of the mouse images.

\section{RESULTS}

\section{Phantom Results for Typical Mouse Imaging Setting}

Because it was the specific aim of this study to characterize the image quality parameters for scan durations and activities typically used in WB mouse experiments in our institute, we first present the results of the phantom scan of 1-h duration and mean activity of 9.4 $\mathrm{MBq}$.

Recovery Coefficients. Figures 1 and 2 show $\mathrm{RC}_{\text {rod }}$ with the corresponding $\% \mathrm{STD}_{\mathrm{RC}}$ of the rods of 1-, 2-, and 3-mm diameter as a function of the number of iterations for different settings of the postreconstruction spatial filter and scatter correction. The effect of no filtering and 3-dimensional gaussian filters with kernel widths of 1.0 and $1.5 \mathrm{~mm}$ in full width at half maximum is demonstrated in Figure 1. These reconstructions were performed without scatter correction. Figure 2 demonstrates the effect of scatter correction. $\mathrm{RC}_{\text {rod }}$ can be more than 1 because of image noise. According to NEMA NU 4, the line profile across which the average activity concentration for the rod has to be determined is the one with the maximum average pixel value. Therefore, for noisy images and rods with a diameter larger than the spatial resolution of the scanner, line profiles exist for which the average pixel value is larger than the mean phantom activity concentration, leading to an $\mathrm{RC}_{\text {rod }}$ that is more than 1 .

Figures 1 and 2 do not show the results for the 4- and 5-mm-diameter rods because full recovery had already been reached at a small number of iterations. For completeness, however, these results have been included in Supplemental Figure 1.

Noise in Uniform Phantom Region. In Figure 3, the effect of postreconstruction filtering on noise in the uniform phantom region as a function of the number of iterations is shown. Scatter correction had no effect on $\% \mathrm{STD}_{\text {unif }}$, and for clarity only the results without scatter correction are shown.

Spillover. SOR for the cold-water and air compartments is shown in Figure 4 as a function of the number of iterations, with and without scatter correction. Postreconstruction filtering did not change the SOR values; for clarity, the results with filtering are not shown. It is seen that $\mathrm{SOR}_{\text {air }}$ with scatter correction still appears to decrease after 6 iterations. We therefore tested whether more iterations would further decrease $\mathrm{SOR}_{\text {air }}$. However, convergence to a value of about 0.045 was reached at 7 iterations.

Because SOR is the ratio between activity concentration measured in the cold compartments and the uniform phantom region, it is interesting to check the influence of scatter correction on the uniform region concentration separately. Scatter correction was found to decrease the mean activity concentration in this region by about $5.9 \%$,
FIGURE 1. $R C_{\text {rod }}(\mathrm{A})$ and $\% \mathrm{STD}_{\mathrm{RC}}(\mathrm{B})$ for 99mTc-filled rods of 1-, 2-, and 3-mm diameter as function of number of iterations showing effect of postreconstruction spatial filtering. Mean activity was $9.4 \mathrm{MBq}$ with 1-h WB acquisition time. All reconstructions were performed without scatter correction. $\mathrm{FWHM}=$ full width at half maximum.

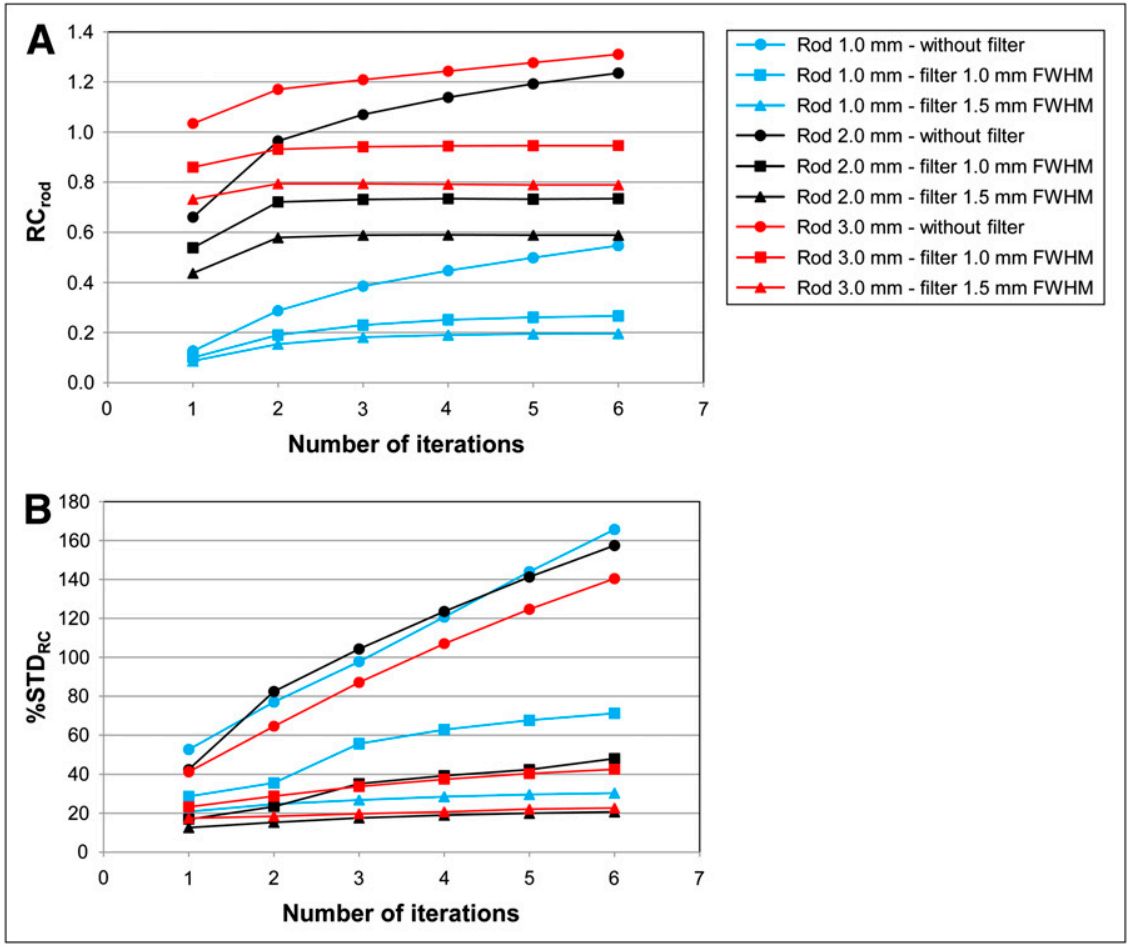




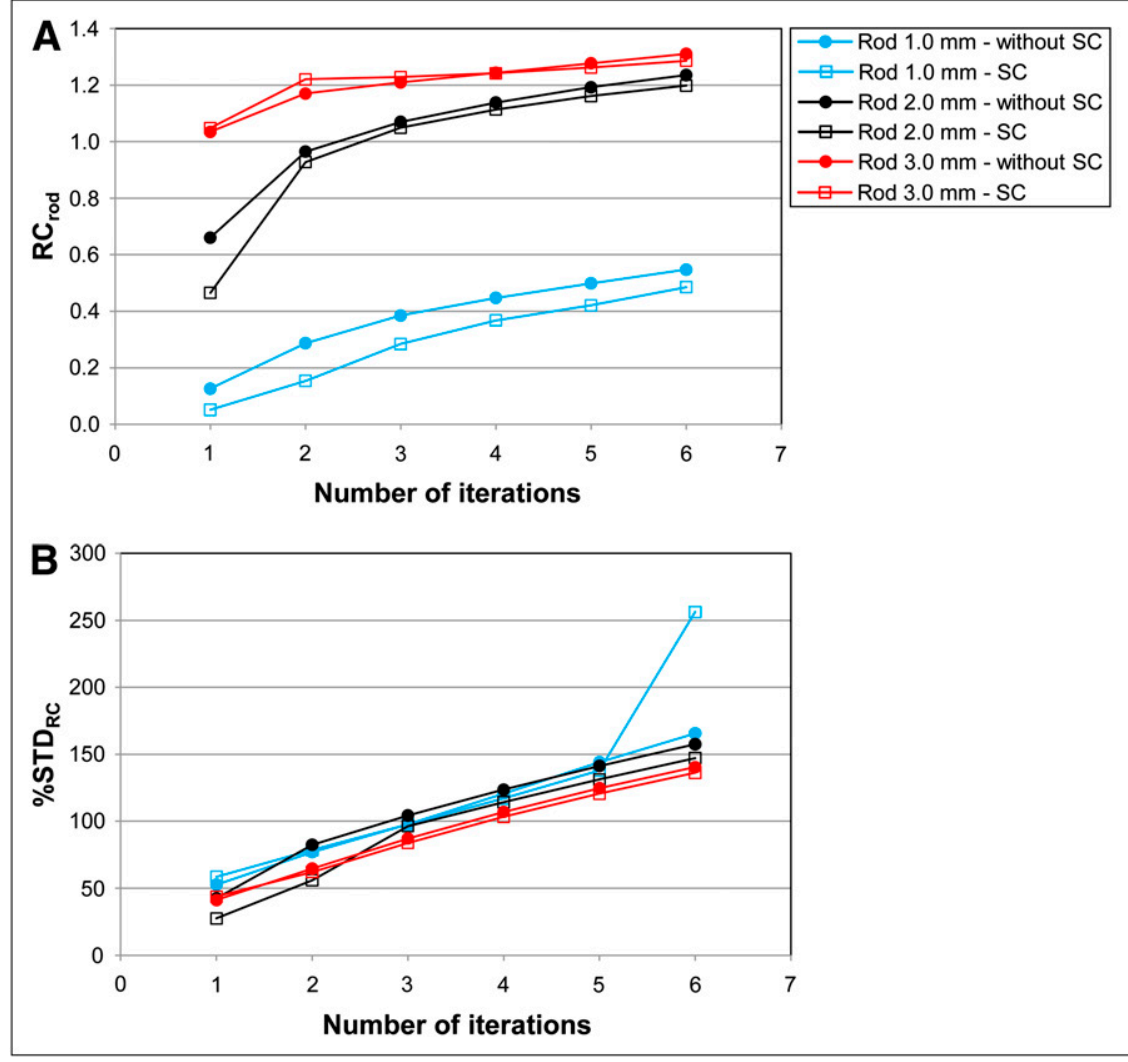

FIGURE 2. $R C_{\text {rod }}(\mathrm{A})$ and $\% \mathrm{STD}_{\mathrm{RC}}(\mathrm{B})$ for 99mTc-filled rods of 1-, 2-, and 3-mm diameter as function of number of iterations showing effect of scatter correction (SC). Mean activity was $9.4 \mathrm{MBq}$ with 1-h WB acquisition time. All reconstructions were performed without postreconstruction filtering. independent of postreconstruction filtering and the number of iterations.

\section{Phantom Results as Function of Activity}

This section describes the image quality parameters for the 20 consecutive WB scans with average activities ranging between 8.4 and $78.2 \mathrm{MBq}$.

Recovery Coefficients. $\mathrm{RC}_{\mathrm{rod}}$ was found to be relatively constant for the whole range of activities. However, for the lowest activities, especially without postreconstruction spatial filtering, the values strongly fluctuated around the average value obtained for the higher activities (Supplemental Fig. 1, upper part of graphs). These fluctuations are in accordance with the large values of $\% \mathrm{STD}_{\mathrm{RC}}$ (even $>$ $100 \%$ ) as shown in Supplemental Figure 1, lower part of graphs. The fluctuations in $\mathrm{RC}_{\mathrm{rod}}$ as a function of activity, and accordingly the corresponding $\% \mathrm{STD}_{\mathrm{RC}}$, decreased when postreconstruction spatial filtering was used.

Noise in Uniform Phantom Region. $\% \mathrm{STD}_{\text {unif }}$ as a function of phantom activity for the different filter settings is shown in Figure 5. The same trend as for $\% \mathrm{STD}_{\mathrm{RC}}$ was observed: $\% \mathrm{STD}_{\text {unif }}$ increased with the number of iterations and decreased with higher activity and spatial filter width.

Spillover. SOR was not affected by the amount of activity. Again, application of postreconstruction filtering did not change SOR (results not shown).

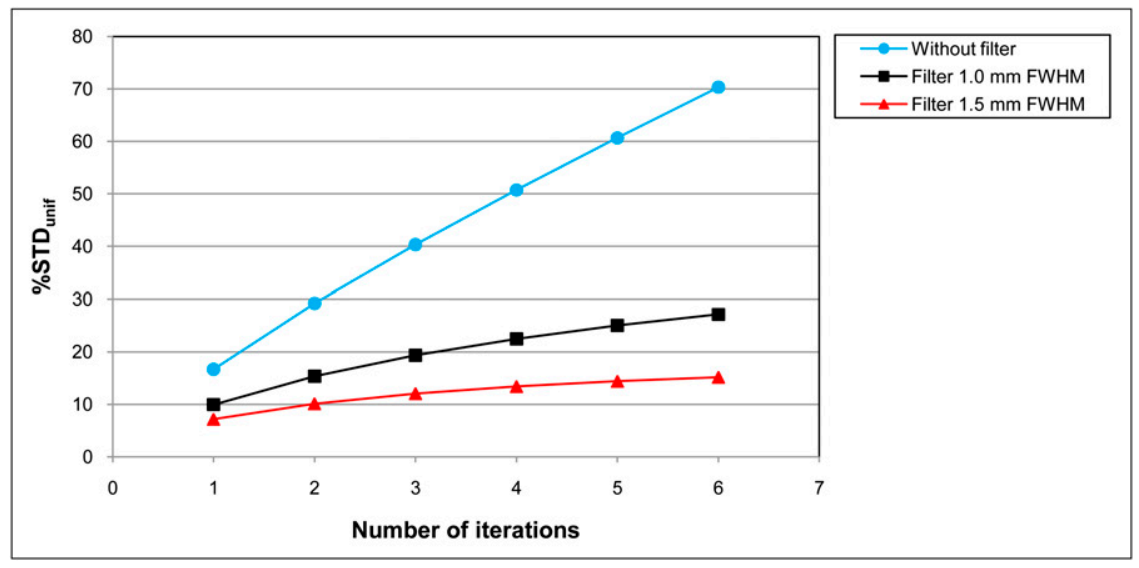

FIGURE 3. \%STD ${ }_{\text {unif }}$ for ${ }^{99 m T c-f i l l e d ~ u n i-~}$ form phantom region as function of number of iterations, with different postreconstruction filter settings. Scatter correction did not change $\% S T D_{\text {unif }}$; accordingly, only results without scatter correction are shown. Mean activity was $9.4 \mathrm{MBq}$ with 1-h WB acquisition time. FWHM = full width at half maximum. 
FIGURE 4. SOR for cold water and air compartments as function of number of iterations, with and without scatter correction (SC). Postreconstruction spatial filtering did not change \%STD unif; accordingly, only results without filtering are shown. Mean activity was $9.4 \mathrm{MBq}$ with 1-h WB acquisition time.

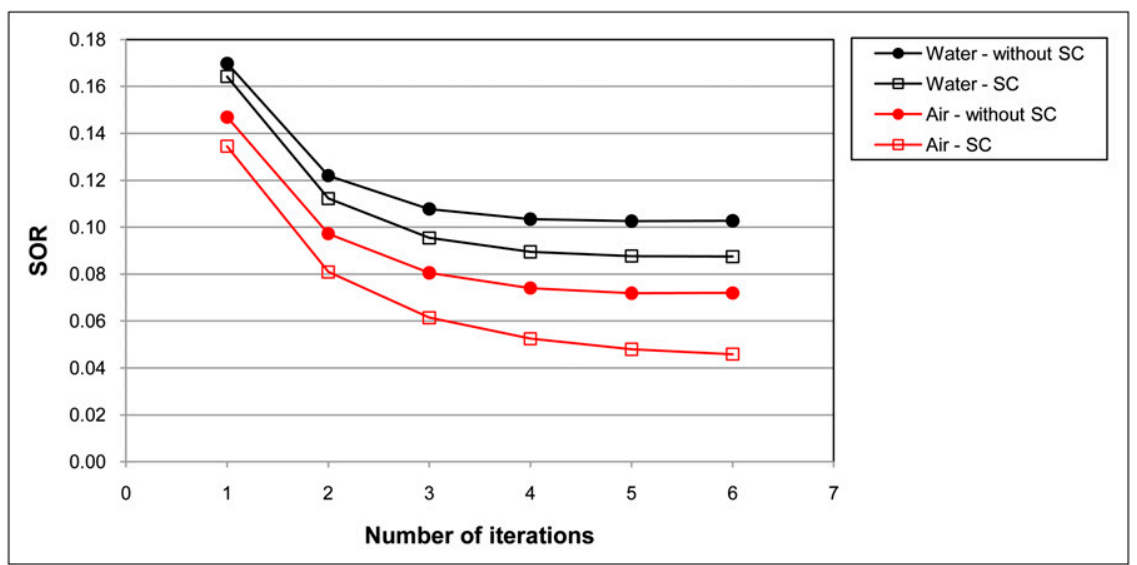

Effective WB Sensitivity. Supplemental Figure 2 shows the average measured counts per second (cps) over all bed positions in the photopeak window as a function of average phantom activity for each time frame. A highly linear relation with $R^{2}=0.99996$ was observed. Effective WB sensitivity (slope of the linear fit) equaled $117 \mathrm{cps} / \mathrm{MBq}$. The offset of 172 cps corresponds to the mean background activity in the laboratory as measured in the photopeak window.

\section{Animal and Phantom Images}

Transverse images of the NU 4 IQ phantom showing the filled rods, the uniform phantom region, and the region containing the cold-water and air compartments are shown in Figures 6A and 6B. Figure 6A was obtained using 6 iterations, scatter correction, and no postreconstruction filtering. Figure 6B shows the effect of postreconstruction filtering with a gaussian filter of $1.0 \mathrm{~mm}$ in full width at half maximum. The ${ }^{99 \mathrm{~m}} \mathrm{Tc}$-hydroxymethylene diphosphonate bone scans of the mouse are shown in Figures 6C and $6 \mathrm{D}$ for the same settings as the phantom images. Supplemental Figures 3 and 4 show images obtained using additional variations of reconstruction settings and amounts of activity for the mouse and phantom, respectively.

\section{DISCUSSION}

The aim of this study was to investigate the usefulness of the NU 4 IQ phantom and parameters to evaluate the image quality performance of a multipinhole small-animal SPECT system.

It was observed that smaller rods need more iterations to obtain maximum $\mathrm{RC}_{\text {rod }}$, but $\% \mathrm{STD}_{\mathrm{RC}}$ increased rapidly as a function of the number of iterations (Fig. 1). Application of postreconstruction spatial filtering reduced $\% \mathrm{STD}_{\mathrm{RC}}$ considerably but also led to a smaller $\mathrm{RC}_{\text {rod }}$. This trade-off between recovery of small structures and image noise, influenced by the number of iterations and spatial filtering, should be considered in the reconstruction settings for small-animal experiments. The optimum settings will of course depend on issues such as the size of the structures to be imaged, scan duration, total activity, and the signal-tobackground ratio for these structures.
Somewhat unexpectedly, for small numbers of iterations, the use of scatter correction led to smaller activity recovery, especially for the 1-mm-diameter rod, and to a smaller extent for the $2 \mathrm{~mm}$-diameter rod, as shown in Figure 2A. In contrast, $\% \mathrm{STD}_{\mathrm{RC}}$ was not influenced using scatter correction, as shown in Figure 2B. The outlier in $\% \mathrm{STD}_{\mathrm{RC}}$ for the 1-mm rod with scatter correction and 6 iterations can be explained by noting that according to NEMA NU 4, the line profile through the rod over which the average activity concentration is calculated is positioned such as to yield maximum total activity along the line. In cases of high noise, the implication is that these profiles will not always be calculated over exactly the same transverse pixel positions. Indeed, for this outlier, the line profile was shifted by 1 pixel $(x)$ and by 2 pixels $(y)$ relative to the profiles of the other data points in Figure 2B.

$\% \mathrm{STD}_{\text {unif }}$ was not affected by scatter correction and shows the same trends as $\% \mathrm{STD}_{\mathrm{RC}}$. $\% \mathrm{STD}_{\text {unif }}$ increases as a function of the number of iterations and decreases when postreconstruction spatial filtering is applied (Fig. 3).

SOR was found to decrease as a function of the number of iterations (Fig. 4), showing that cold regions in hot environments can be recovered only by using many iterations, as has previously been described for PET image reconstruction (17). Furthermore, $\mathrm{SOR}_{\text {wat }}$ was much larger than $\mathrm{SOR}_{\text {air }}$ - $\mathrm{a}$ finding that is clearly attributed to the fact that photon scatter in water is stronger than in air. It is interesting to note the differences in scatter-related spillover effects between SPECT and PET. Related to the detection of photon pairs forming lines of response in PET, spillover is to a large extent caused by scatter outside the cold compartments, causing the corresponding lines of response to be falsely attributed to these compartments, independently of their density and scatter efficiency. This observation was made in small-animal ${ }^{18} \mathrm{~F}$-PET (18), leading to almost equal values for $\mathrm{SOR}_{\text {air }}$ and $\mathrm{SOR}_{\mathrm{wat}}$. Because of singlephoton detection in SPECT, however, the activity measured in cold regions should ideally be proportional to the residual photon scatter originating in the cold region itself, as explains the observed difference between $\mathrm{SOR}_{\text {air }}$ and $\mathrm{SOR}_{\mathrm{wat}}$. Scatter correction had a positive effect on image 


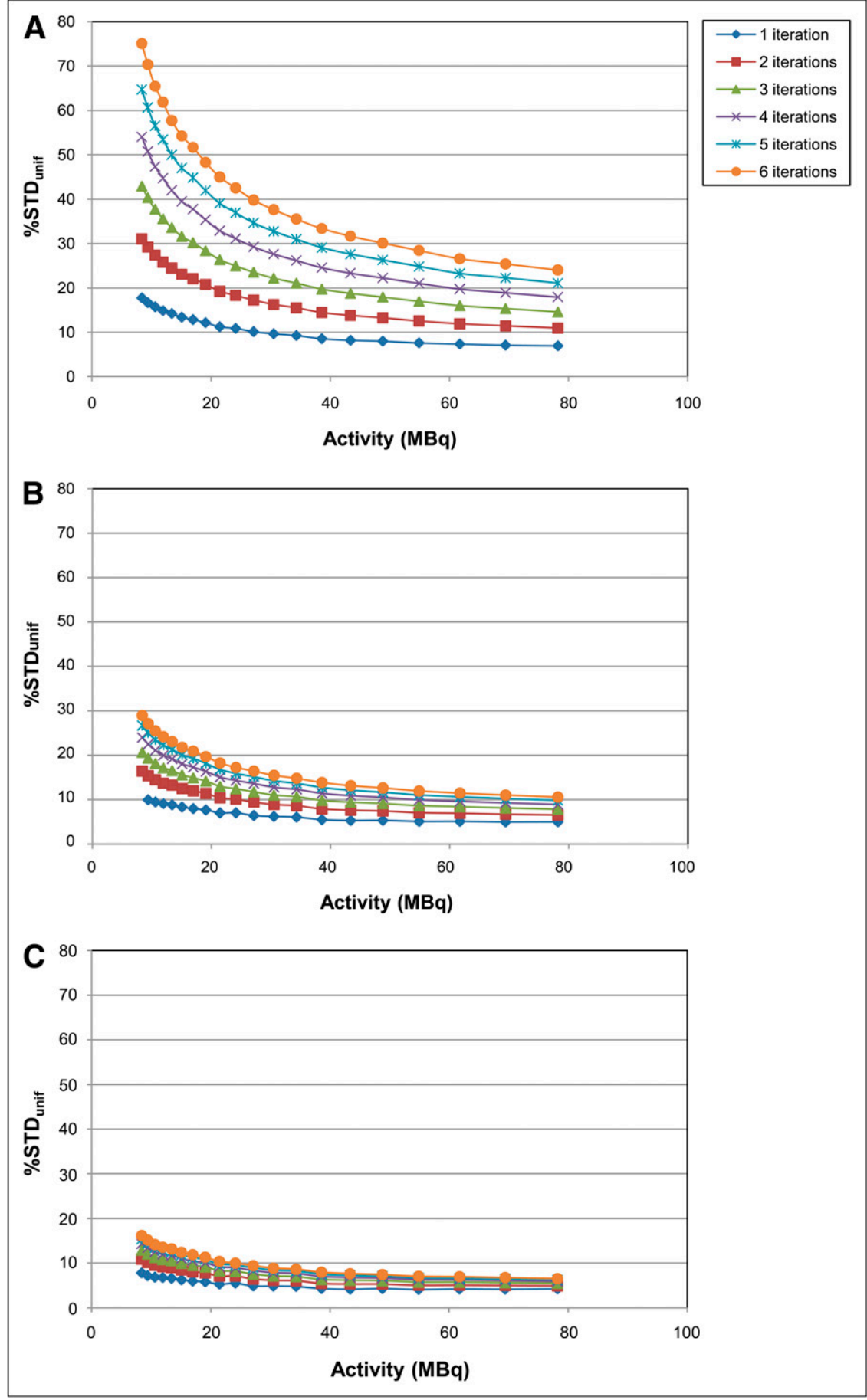

FIGURE 5. \%STD unif as function of average phantom activity for time frames of 1 -h WB acquisition time: unfiltered $(A)$ and postreconstruction gaussian filter of $1.0 \mathrm{~mm}(B)$ and $1.5 \mathrm{~mm}$ in full width at half maximum (C). quality because SOR decreased, for both the water-filled and the air-filled compartments. It is, however, unknown why this decrease was larger for air than for water. A detailed comparison of the present results with those for a state-of-the art small-animal PET scanner can be made using information provided by Visser et al. (19).

The effect of the activity level was especially seen in the image noise, which increased rapidly for decreasing activity (Fig. 5). On the other hand, $\mathrm{RC}_{\text {rod }}$ and SOR did not systematically change as a function of activity, although these parameters were fluctuating around their average value for lower activities (Supplemental Fig. 1 shows data for $\mathrm{RC}_{\mathrm{rod}}$ ).

The scan duration of $1 \mathrm{~h}$ and activity of $10 \mathrm{MBq}$, considered typical for WB SPECT mouse scans, are larger than the values prescribed for small-animal NEMA PET (20 min and $3.7 \mathrm{MBq}$ of ${ }^{18} \mathrm{~F}$, respectively) because WB SPECT has lower sensitivity than WB PET. Shorter scan times and lower activity, however, could be used when scanning isolated organs or tumors because reducing the 
A
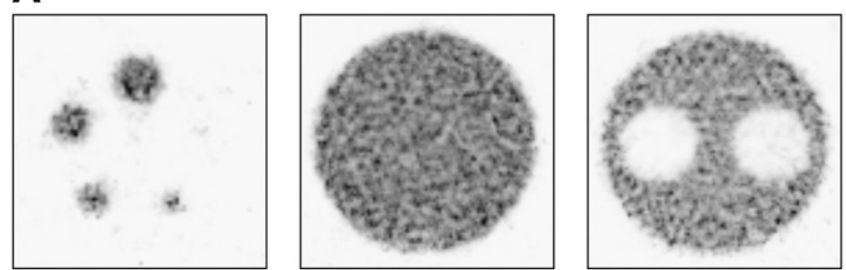

C

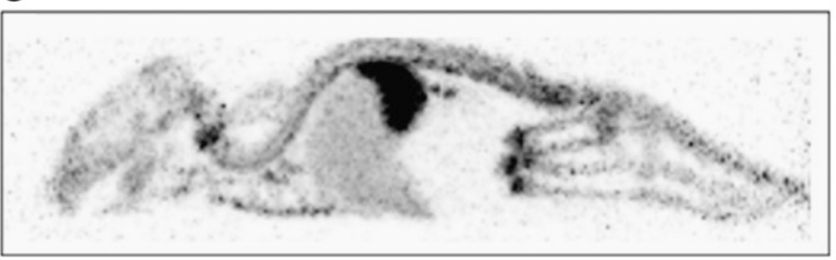

B
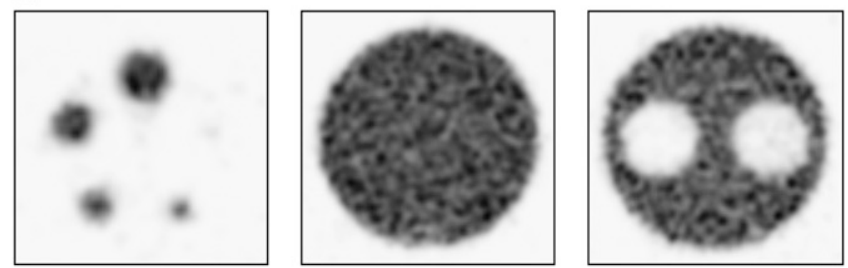

D

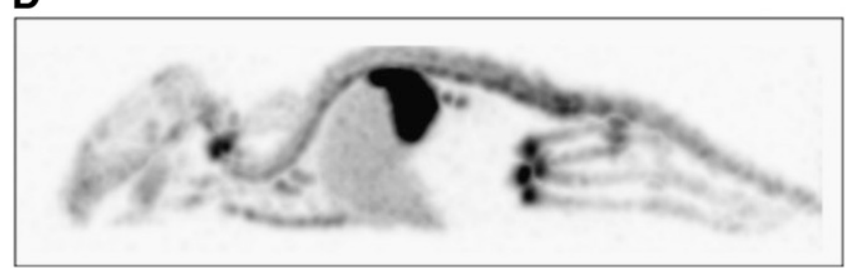

FIGURE 6. (A and B) Transverse images of NU 4 IQ phantom showing, from left to right, filled rods, uniform phantom region, and region containing cold water and air compartments. Images were obtained using $9.4 \mathrm{MBq}$, scatter correction, no filter, and 6 iterations (A) or 9.4 $\mathrm{MBq}$, scatter correction, postreconstruction gaussian filter of $1.0 \mathrm{~mm}$ in full width at half maximum, and 6 iterations (B). (C and D) Maximumintensity-projection images of 99mTc-hydroxymethylene diphosphonate bone scans of mouse. WB acquisition time was $1 \mathrm{~h}$ in all cases. Images were obtained using $9.4 \mathrm{MBq}$, scatter correction, no filter, and 6 iterations (C) or $9.4 \mathrm{MBq}$, scatter correction, postreconstruction gaussian filter of $1.0 \mathrm{~mm}$ in full width at half maximum, and 6 iterations (D).

field of view in multipinhole SPECT generally increases effective sensitivity.

As expected, the effective WB sensitivity of $117 \mathrm{cps} /$ $\mathrm{MBq}$ is smaller than the centered-point-source sensitivity of $700 \mathrm{cps} / \mathrm{MBq}$ as presented elsewhere (2). The difference between these values reflects the combined effect of photon attenuation and partial coverage of the total phantom activity in individual bed positions. Effective WB sensitivity is related to the choice of phantom. The NU 4 IQ phantom clearly is somewhat larger than a typical mouse but much smaller than a rat. Instead, one could also use the NEMA NU 42008 counting rate and scatter fraction phantoms (radioactive line source in cold polyethylene cylinder (3)), for which differently sized mouse- and ratlike sizes have been defined.

The phantom experiments provided quantitative and objective data on the effect of different reconstruction and acquisition settings for a range of activity levels. Depending on the specific imaging goals, these image quality parameters can guide the researcher in the choice for optimum imaging settings in real small-animal experiments, for example, smoothing of images with low noise levels, suppression of scattered photons for better quantification, or detection of small organ structures. As such, the WB mouse scans (Fig. 6 and Supplemental Fig. 3) illustrated the difference between the various settings resulting in how much detail can be distinguished and the amount of image noise. Especially for smaller structures such as the small bony components of the sternum or the ribs, the differences were clearly visualized. The same characteristics as in these mouse images can be noted in the phantom images of Figure 6 and Supplemental Figure 4.

The U-SPECT-II scanner can be equipped with different collimators. Besides the one used in the present study (pinhole and bore diameter of 1.0 and $98 \mathrm{~mm}$, respectively), collimators with pinhole diameters of 0.35 and $0.6 \mathrm{~mm}$ and a bore diameter of $44 \mathrm{~mm}$ are commercially available (2). However, because of the use of scanning-focus mode, the maximum diameter of objects to be completely scanned in these collimators is $28 \mathrm{~mm}$, prohibiting complete WB scans of the NU 4 IQ phantom (outer diameter, $33.5 \mathrm{~mm}$ ) since the outer part of the phantom would collide with the collimator wall when the opposite side is moved into the center of the field of view.

The present paper has dealt with the image quality aspect of small-animal SPECT. In a broader sense, one could discuss how a future NEMA "NU 5" standard for SPECT should be defined and to what extent it would differ from NEMA NU 4 for PET. In short, we believe that image quality parameters could be defined in the same way as for PET, and the NU 4 IQ phantom would be appropriate for WB mouse or rat scans. However, for scanning in highspatial-resolution mode-for example, single-organ or single-tumor imaging-it should be discussed whether an additional phantom with smaller internal structures and smaller outer diameter would be appropriate.

Spatial resolution as defined by NEMA NU 4 (point source, reconstructed using filtered backprojection) cannot easily be adapted to multipinhole SPECT, because the iterative reconstruction algorithms used do not yield univocal results for point or line sources, and filtered backprojection is normally not available. Therefore, spatial resolution expressed as recovery coefficients for small rods in an image quality phantom would be more appropriate. However, because recovery coefficients depend on reconstruction settings, trade-offs with, for example, noise and spillover should be considered. For comparison of different vendors or collimators, the accompanying image quality parameters should be specified along with the recovery coefficients. 
Count losses and scatter fraction could be measured in multipinhole SPECT using the same (mouselike and ratlike) phantoms as in NEMA NU 4. However, we have found that count loss is not of much importance in view of the highly linear relation between counting rate and source activity, even for high activities (Supplemental Fig. 2).

Finally, as already mentioned, sensitivity measured using a point source as in NEMA NU 4 is not appropriate for multipinhole SPECT. Instead, standardized, larger phantoms should be defined.

\section{CONCLUSION}

Although developed for small-animal PET, the NU 4 IQ phantom was found to be useful for small-animal SPECT as well, allowing for quantitative determination of image quality parameters. The U-SPECT-II scanner with a 1.0$\mathrm{mm}$ pinhole collimator was used to demonstrate the effect of the different image quality parameters for a range of activity levels and different reconstruction settings. Tradeoffs between spatial resolution, image noise, and scatter performance have been quantified and related to acquisition and image reconstruction settings and illustrated in a WB, ${ }^{99 \mathrm{~m}} \mathrm{Tc}$-hydroxymethylene diphosphonate mouse scan.

\section{ACKNOWLEDGMENT}

No potential conflict of interest relevant to this article was reported.

\section{REFERENCES}

1. Meikle SR, Kench P, Kassiou M, Banati RB. Small animal SPECT and its place in the matrix of molecular imaging technologies. Phys Med Biol. 2005;50:R45R61.

2. van der Have F, Vastenhouw B, Ramakers RM, et al. U-SPECT-II: an ultra-highresolution device for molecular small-animal imaging. J Nucl Med. 2009; 50:599-605.
3. NEMA Standards Publication NU 4-2008: Performance Measurements of Small Animal Positron Emission Tomographs. Rosslyn, VA: National Electrical Manufacturers Association; 2008.

4. Beekman FJ, van der Have F, Vastenhouw B, et al. U-SPECT-I: a novel system for submillimeter-resolution tomography with radiolabeled molecules in mice. J Nucl Med. 2005;46:1194-1200.

5. Difilippo FP. Design and performance of a multi-pinhole collimation device for small animal imaging with clinical SPECT and SPECT-CT scanners. Phys Med Biol. 2008;53:4185-4201.

6. Metzler SD, Vemulapalli S, Jaszczak RJ, Akabani G, Chin BB. Feasibility of whole-body functional mouse imaging using helical pinhole SPECT. Mol Imaging Biol. 2010;12:35-41.

7. Ogawa K, Ohmura N, Iida H, Nakamura K, Nakahara T, Kubo A. Development of an ultra-high resolution SPECT system with a CdTe semiconductor detector. Ann Nucl Med. 2009;23:763-770.

8. Schramm NU, Ebel G, Engeland U, Schurrat T, Behe M, Behr TM. High-resolution SPECT using multipinhole collimation. IEEE Trans Nucl Sci. 2003;50:315-320.

9. Lackas C, Schramm NU, Hoppin JW, Engeland U, Wirrwar A, Halling H. TSPECT: a novel imaging technique for small animal research. IEEE Trans Nucl Sci. 2005;52:181-187.

10. Beekman FJ, Vastenhouw B, van der Wilt G, et al. 3-D rat brain phantom for high-resolution molecular imaging. Proc IEEE. 2009;97:1997-2005.

11. Difilippo FP, Gallo SL, Klatte RS, Patel S. A fillable micro-hollow sphere lesion detection phantom using superposition. Phys Med Biol. 2010;55:5363-5381.

12. Park MA, Zimmerman RE, Taberner A, Kaye MW, Moore SC. Design and fabrication of phantoms using stereolithography for small-animal imaging systems. Mol Imaging Biol. 2008;10:231-236.

13. van der Have F, Vastenhouw B, Rentmeester M, Beekman FJ. System calibration and statistical image reconstruction for ultra-high resolution stationary pinhole SPECT. IEEE Trans Med Imaging. 2008;27:960-971.

14. Vastenhouw B, Beekman F. Submillimeter total-body murine imaging with USPECT-I. J Nucl Med. 2007;48:487-493.

15. Branderhorst W, Vastenhouw B, Beekman FJ. Pixel-based subsets for rapid multi-pinhole SPECT reconstruction. Phys Med Biol. 2010;55:2023-2034.

16. Ogawa K, Harata Y, Ichihara T, Kubo A, Hashimoto S. A practical method for position-dependent Compton-scatter correction in single photon emission CT. IEEE Trans Med Imaging. 1991;10:408-412.

17. Boellaard R, van Lingen A, Lammertsma AA. Experimental and clinical evaluation of iterative reconstruction (OSEM) in dynamic PET: quantitative characteristics and effects on kinetic modeling. J Nucl Med. 2001;42:808-817.

18. Disselhorst JA, Brom M, Laverman P, et al. Image-quality assessment for several positron emitters using the NEMA NU 4-2008 standards in the Siemens Inveon small-animal PET scanner. J Nucl Med. 2010;51:610-617.

19. Visser EP, Disselhorst JA, van Lier MGJTB, et al. Characterization and optimization of image quality as a function of reconstruction algorithms and parameter settings in a Siemens Inveon small-animal PET scanner using the NEMA NU 42008 standards. Nucl Instr Meth A. 2011;629:357-367. 